\title{
Equilibrium Sorption of Structurally Diverse Organic Ions to Bovine Serum Albumin
}

\section{Luise Henneberger, Kai-Uwe Goss, and Satoshi Endo}

\begin{tabular}{|c|l|}
\hline Citation & Environmental Science \& Technology, 50(10); 5119-5126 \\
\hline Issue Date & $2016-04-20$ \\
\hline Type & Journal Article \\
\hline Textversion & author \\
\hline Right & $\begin{array}{l}\text { This document is the Accepted Manuscript version of a Published Work that appeared in } \\
\text { final form in Environmental Science \& Technology, copyright @ American Chemical Society } \\
\text { after peer review and technical editing by the publisher. To access the final edited and } \\
\text { published work see https://doi.org/10.1021/acs.est.5b06176 }\end{array}$ \\
\hline URI & http://dlisv03.media.osaka-cu.ac.jp/il/meta_pub/G0000438repository_15205851-50-10-5119 \\
\hline DOI & $10.1021 / a c s . e s t .7 b 05144$ \\
\hline
\end{tabular}

SURE: Osaka City University Repository http://dlisv03.media.osaka-cu.ac.jp/il/meta_pub/G0000438repository 
$4 \uparrow \dagger$ Helmholtz Centre for Environmental Research UFZ, Permoserstr. 15, D-04318 Leipzig, Germany

$\$$ University of Halle-Wittenberg, Institute of Chemistry, Kurt-Mothes-Str. 2, D-06120 Halle, Germany

\section{ABSTRACT}

11 Reliable partitioning data are essential for assessing the bioaccumulation potential and the

12 toxicity of chemicals. In contrast to neutral organic chemicals, the partitioning behavior of

13 ionogenic organic chemicals (IOCs) is still a black box for environmental scientists. Partitioning

14 to serum albumin, the major protein in blood plasma, strongly influences the freely dissolved

15 concentration of many chemicals (including IOCs), which affects their transport and distribution

16 in the body. Because consistent datasets for partitioning of IOCs are rarely available, bovine

17 serum albumin-water partition coefficients $\left(K_{\mathrm{BSA} / \mathrm{w}}\right)$ were measured in this study for 45 anionic 
18 and 4 cationic organic chemicals, including various substituted benzoic and naphthoic acids,

19 sulfonates and several pesticides and pharmaceuticals. The results of this study suggest that

20 binding to BSA is substantially influenced by the three dimensional structure of the chemicals

21 and the position of substitutions on the sorbing molecules. For example, we found a difference of

$22>1.5 \log$ units between isomeric chemicals such as 3,4-dichlorobenzoic acid and 2,6-

23 dichlorobenzoic acid, 1-naphthoic acid and 2-naphthoic acid, and 2,4,6-trimethylbenzoic acid

24 and 2,4,6-trimethylbenzenesulfonate. Conventional modeling approaches (e.g. based on octanol-

25 water partition coefficients) poorly predict $\log K_{\mathrm{BSA} / \mathrm{w}}$ of organic ions $\left(R^{2} \leq 0.5\right)$, partially

26 because they do not capture the observed steric effects. Hence, alternative modeling strategies

27 will be required for accurate prediction of serum albumin-water partition coefficients of organic 28 ions.

\section{Introduction}

31 Partitioning of ionogenic organic chemicals (IOCs) into biological tissues and their

32 constituents such as various lipids and proteins is of great importance, as it has direct

33 implications for bioconcentration, bioaccumulation, and toxicity of IOCs. However, established

34 models to predict relevant partition coefficients are almost exclusively focused on neutral

35 organic chemicals including the neutral species of ionizable chemicals. For example, a simple

36 regression with $\log K_{\mathrm{ow}}$ is widely used as a screening model, which sometimes gives a

37 surprisingly good approximation (e.g., for membrane-water partition coefficients). ${ }^{1}$

38 Polyparameter linear free energy relationships (PP-LFERs) can give more accurate predictions

39 for broader ranges of chemicals and partition phases. ${ }^{2,3}$ These empirical models are convenient

40 but applicable only for neutral chemicals. To our knowledge, there is only one model that was 
41 developed specifically for the prediction of biopartitioning of IOCs in general. ${ }^{4}$ One reason for

42 the absence of models may be the lack of experimental data that are measured under consistent

43 conditions for partitioning of charged chemicals.

44 Bioaccumulation models used for risk assessment often assume that the dominant sorption 45 phase in organisms is the total lipid fraction. For IOCs storage lipids are expected to play a minor 46 role while phospholipid membranes and the protein fraction of an organism are supposed to 47 contribute significantly to the overall partitioning process. ${ }^{5}$ Serum albumin, the most abundant 48 protein in blood plasma, is likely an important sorption phase for IOCs, because research on 49 pharmaceuticals and endogenous chemicals has long shown that serum albumin binds a broad 50 spectrum of chemicals, especially hydrophobic anions of medium size ${ }^{6-8}$ Binding to serum 51 albumin has major impact on transport and distribution of a target chemical in the body, because 52 it increases the sorption capacity of the blood and decreases the free, unbound concentration of 53 the chemical. ${ }^{9}{ }^{10}$ Moreover, fetal bovine serum is widely used for cell culture assays, where 54 serum albumin binding often determines the bound and the unbound fractions of the test 55 chemical in the medium. ${ }^{11}$ Furthermore, serum albumin is often considered a generic protein to 56 represent various properties of the bulk protein fraction of organisms, including sorption 57 properties, ${ }^{12}$ although this assumption may not always be valid and thus needs careful 58 evaluation. ${ }^{13}$

59 In the literature, binding of a target chemical to protein, e.g., bovine serum albumin (BSA), is 60 typically reported as association constant $K_{\mathrm{a}}\left[\mathrm{M}^{-1}\right]$. For 1:1 binding $K_{\mathrm{a}}$ is defined as:

$61 \quad K_{a}=\frac{C_{\text {bound }}}{C_{\text {free }}[B S A]}$ 
62 where $C_{\text {free }}\left[\mathrm{mol} / \mathrm{L}_{\text {water }}\right]$ is the freely dissolved molar concentration of the chemical, $C_{\text {bound }}$ the

63 molar concentration of the chemical bound to BSA, and [BSA] the free molar concentration of

64 BSA in the solution. The extent of binding to BSA can also be expressed as BSA-water partition

65 coefficient $\left(K_{\mathrm{BSA} / \mathrm{w}}\left[\mathrm{L}_{\text {water }} / \mathrm{kg}_{\mathrm{BSA}}\right]\right)$, which is preferably used by environmental scientists and is

66 defined as the ratio of the concentration of the target chemical in BSA $\left(C_{\mathrm{BSA}}\left[\mathrm{mol} / \mathrm{kg}_{\mathrm{BSA}}\right]\right)$ to the

67 freely dissolved concentration of the chemical:

$68 K_{\mathrm{BSA} / \mathrm{w}}=C_{\mathrm{BSA}} / C_{\mathrm{free}}$

69 In case the chemical concentration is so low that BSA binding is far below saturation, $K_{\mathrm{a}}$ and

$70 K_{\mathrm{BSA} / \mathrm{w}}$ are convertible with a constant factor (i.e., $K_{\mathrm{a}}=10^{1.83} K_{\mathrm{BSA} / \mathrm{w}}$ ). ${ }^{14}$

71 Large amounts of data are available in the literature for binding of pharmaceuticals (including

72 also IOCs) to human serum albumin. However, reliability and comparability of these data are

73 difficult to evaluate. As reviewed by Nilsson et al., ${ }^{15}$ data for protein binding are often published

74 without carefully controlling all the factors that can influence the partitioning process, e.g.,

75 temperature; the $\mathrm{pH}$ value; concentrations of the test chemical, salt, and the protein; non-specific

76 adsorption to the used equipment; equilibrium disturbances; and leaking of dialysis membrane.

77 Moreover, pharmaceuticals are often multifunctional molecules with complex structure. A

78 collection of data for such diverse chemicals is difficult to interpret in terms of structural

79 influences on the binding constant. Systematically measured data for a series of chemicals with

80 incremental changes in the substructure units (e.g., the number of $\mathrm{Cl}$ on the benzene ring) or data

81 for a pair of chemicals that differ by only one structural feature may shed additional light on the

82 binding mechanisms of IOCs to serum albumin.

83 This study aims to elucidate how the molecular structure of organic ions (e.g., different basic

84 structures, substitutions and charged functional groups) influences their partitioning to serum 
85 albumin and which mechanisms underlie the sorption process. To this end, we investigated the

86 partitioning of systematically selected organic anions and cations to BSA, starting from simple

87 compounds onwards to more complex structures. Particularly, many varyingly substituted

88 benzenes and naphthalenes with an anionic functional group were included to study the

89 influences of molecule structures on serum albumin binding. All measurement conditions were

90 thoroughly controlled. Additionally, influence of $\mathrm{pH}$ value, dependence on the concentration of

91 inorganic ions, and reversibility of BSA binding were determined experimentally. Finally, the

92 data obtained were correlated with various descriptors to gain an insight into the requirements of

93 successful modeling for serum albumin binding.

94 Materials and methods

95 Materials

96 Bovine serum albumin (essentially fatty acid free) was purchased from Sigma Aldrich (Product

97 No. A3803) and used without further purification. BSA was chosen because of its good

98 availability and comparability to the previous publication for neutral compounds. ${ }^{14}$ Water

99 purified with a Milli-Q Gradient A10 system from Millipore was used. Methanol (Suprasolv)

100 was obtained from Merck and acetonitrile (gradient grade) from Sigma Aldrich. Unless

101 otherwise noted below, all sorption experiments were performed using Hanks' balanced salt

102 solution (HBSS, without phenol red and sodium bicarbonate, Sigma Aldrich) buffered with 10

$103 \mathrm{mM}$ tris(hydroxymethyl)aminomethane (Tris) from Carl Roth. After addition of sodium

104 bicarbonate and Tris, the $\mathrm{pH}$ value of HBSS was adjusted to 7.4 using $1 \mathrm{~N} \mathrm{HCl}$ or $\mathrm{NaOH}$

105 solution from Merck. The exact salt concentrations of HBSS and a comparison with human

106 plasma are shown in Table S3, SI. Ammonium acetate, formic acid, orthophosphoric acid, bis(2-

107 hydroxyethyl)amino-tris(hydroxymethyl)methane (Bis-Tris), sodium bicarbonate, sodium 
108 chloride, sodium sulfate and sodium azide were purchased from Sigma Aldrich, Carl Roth or 109 Merck.

110 The chemicals used for the binding experiments were different organic acids and bases, or salts

111 of them, all of which have only one ionizable functional group and are more than $99 \%$ ionized at

$112 \mathrm{pH}$ 7.4. To investigate the influence of different substitutions on the binding constant, a series of

113 benzoic acids, naphthoic acids and sulfonates were chosen for the dataset. More complex

114 compounds (i.e., pesticides and pharmaceuticals) were included, because of their environmental

115 relevance. All test chemicals are listed in Table 1 and had purity of at least $98 \%$. More details on

116 the test chemicals (e.g., CAS number, provider, analytical method used for quantification,

117 chemical structure, $\mathrm{p} K_{\mathrm{a}}$ values and recovery from control experiments) can be found in the

118 Supporting Information Table S1. In this work, weak acids and bases are always denoted with

119 the chemical names of their neutral species (e.g., 4-chlorobenzoic acid) because they are more

120 common than the names for the ionic species (e.g., 4-chlorobenzoate), despite the fact that weak

121 acids and bases in our test solutions are always predominantly in their ionic form and that $K_{\mathrm{BSA} / \mathrm{w}}$

122 reported in this work is thus for the ionic species.

\section{Dialysis experiments}

124 Our experimental procedure for the dialysis experiments has been described previously in 125 detail. ${ }^{16}$ In short: a custom-made dialysis cell that consists of two half-cells (total volume approx. $12610 \mathrm{~mL}$ ) and dialysis membranes from Spectrum Laboratories (type Spectra/Por $4 \mathrm{RC}$ with a 127 molecular weight cutoff of $12-14 \mathrm{kD}$ ) was used. One half-cell of the dialysis unit received $5 \mathrm{~mL}$

128 HBSS buffer and the other half-cell $4.9 \mathrm{~mL}$ BSA solution (1-50 g/L). All samples were spiked 129 with $100 \mu \mathrm{L}$ of a dilution of the test chemical in HBSS, which was prepared from a concentrated 130 stock solution in methanol (final concentration of methanol in the system $\leq 0.5 \mathrm{vol} \%$ ), and the 
131 dialysis cells were equilibrated at $37^{\circ} \mathrm{C}$. Three to four replicates were prepared. Aliquots of 100

$132 \mu \mathrm{L}$ were taken from the buffer side of the BSA samples after two and three days (with no

133 significant difference observed between the two time points). The concentration of the test

134 chemicals $\left(C_{\text {free}}\right)$ was quantified in all samples as described in the instrumental analysis section,

$135 C_{\mathrm{BSA}}$ was obtained from the mass balance calculation, and $\log K_{\mathrm{BSA} / \mathrm{w}}$ was calculated using eq 2.

136 Test chemicals for which the fraction bound to BSA was less than $20 \%$ were excluded from the

137 dataset. At high concentrations BSA possibly causes a colloid osmotic pressure that leads to a

138 volume shift in the dialysis cell. However, this was not observed in our experiments. In

139 preliminary experiments we also determined the amount of proteins that passes the dialysis

140 membrane using the Bradford assay. Only $0.01 \%$ of the total protein were found to diffuse

141 through the membrane, which is not expected to influence the determination of $K_{\mathrm{BSA} / \mathrm{w}}$ in our

142 experiments. Control samples without BSA were also prepared and measured in parallel. If the

143 recovery for a test chemical from the control was consistently below $95 \%$ or above $105 \%$, the

144 concentration in the BSA samples was corrected according to the recovery. This correction is

145 justified, because a consistent deviation from $100 \%$ recovery was found to result from the first

146 dilution step of the methanolic stock solution in HBSS, which should cause exactly the same

147 error in the dose amounts for BSA and control samples. Test chemicals with recoveries below 90

148 and above $120 \%$ were excluded from the dataset. The average recovery from the control

149 samples for the remaining test chemicals was 91-117\%. All sorption experiments were

150 performed for individual chemicals and not with mixtures. Additionally, in all experiments the

151 amount of the bound test chemicals was kept well below the amount of BSA (i.e., $\leq 0.1 \mathrm{~mol} / \mathrm{mol}$

152 at equilibrium, see also Table S2, SI), to avoid saturation of the binding sites of BSA. More

153 details on the dialysis experiments are listed in Table S2, SI (e.g., concentration of stock 
154 solutions in methanol, initial water phase and measured equilibrium water phase concentrations

155 of all test chemicals, concentration of BSA solution used for the dialysis experiments).

\section{Reversibility of BSA binding}

157 To test the reversibility of binding and the mass conservation, the following experiment was

158 performed for a subset of the test chemicals. Dialysis cells with BSA were prepared as described

159 above, but additionally with $300 \mathrm{mg} / \mathrm{L}$ sodium azide to prevent microbial activity. This was

160 necessary to extend the experimental time without causing precipitation of BSA. Additional

161 experiments showed no significant influence of sodium azide on the partitioning of benzoic acid,

162 2-phenoxyacetic acid and 2-methoxy-1-naphthoic acid to BSA (data not shown). After

163 equilibrium was established (three days) the buffer-containing half-cell was emptied completely

164 and $5 \mathrm{~mL}$ of fresh buffer were added. Additional three days were given for re-equilibration and

165 the buffer was sampled again. With both equilibrium buffer concentrations after three and six

166 days, $\log K_{\mathrm{BSA} / \mathrm{w}}$ was calculated, assuming the mass conservation. For samples taken after six

167 days, the removal of test chemical due to clearance of buffer after three days was considered in

168 the mass balance calculation. If binding to BSA was fully reversible and no mass loss occurred,

169 the determined $K_{\mathrm{BSA} / \mathrm{w}}$ after three and six days should be the same. If either (or both) of the

170 conditions is not fulfilled, $K_{\mathrm{BSA} / \mathrm{w}}$ calculated for the six days sample should become larger than

171 that for the three days sample.

\section{Dependence of BSA binding on $\mathbf{p H}$ value and salt concentration}

173 The partitioning of IOCs to proteins may be influenced by $\mathrm{pH}$ value and salt concentration. In

174 this study sorption of 2,6-dichlorobenzoic acid to BSA was measured at $\mathrm{pH} 6,7$ and 8 at a 175 constant concentration of $\mathrm{Cl}^{-}(150 \mathrm{mM})$. To control the $\mathrm{pH}$ value in the experiments, $10 \mathrm{mM}$ Bis-

176 Tris $\left(\mathrm{p} K_{\mathrm{a}}=6.5\right)$ were added for solutions at $\mathrm{pH} 6$ and $10 \mathrm{mM}$ Tris $\left(\mathrm{p} K_{\mathrm{a}}=8.06\right)$ for $\mathrm{pH} 7$ and 8 
177 (for more details see SI). Salt concentration dependence was investigated by measuring the BSA178 water partition coefficient of 2,6-dichlorobenzoic acid at $\mathrm{Cl}^{-}$concentrations of 10, 50, 300 and $179500 \mathrm{mM}$ (adjusted with $\mathrm{NaCl}$ ) and at a $\mathrm{SO}_{4}{ }^{2-}$ concentration of $163 \mathrm{mM}$ (added as $\mathrm{Na}_{2} \mathrm{SO}_{4}$; the 180 same ionic strength as $500 \mathrm{mM} \mathrm{Cl}^{-}$). These salt solutions contained $10 \mathrm{mM}$ Tris and $10 \mathrm{mM} \mathrm{HCl}$ 181 and $\mathrm{pH}$ was adjusted to 7 with $0.1 \mathrm{~N} \mathrm{NaOH}$ solution.

\section{Instrumental analysis}

183 For the majority of the test chemicals an HPLC system from JASCO was used, equipped with 184 either a UV detector (UV-970 M, JASCO) or a fluorescence detector (RF-10AXL, Shimadzu). 185 For chemicals that needed a sensitive quantification method, LC-MS/MS measurements were 186 performed with two different instruments: an Acquity UPLC system from Waters with a Xevo 187 TQ mass spectrometer and an UPLC system from Agilent Technologies (1290 Infinity Series) 188 equipped with a 6400 Triple Quad mass spectrometer. Details on the instrumental analysis are 189 presented in the Supporting information.

\section{Results and discussion}

\section{Reversibility tests}

192 The results from the reversibility tests with BSA performed for eight test chemicals are 193 presented in Table S6, SI. No significant difference between the partition coefficients determined 194 after three and six days was found (difference between the mean values was $<0.03 \log$ units for 195 all chemicals), indicating that binding to BSA is a fully reversible process and that there was no 196 significant mass loss for the chemicals tested. For all other test chemicals for which we report the 197 partition coefficients in this study, we, therefore, assume that the interaction with BSA is non198 covalent and, in principle, reversible. It is reasonable to think that usual sorption of organic 
199 chemicals to BSA is reversible, because, otherwise, serum albumin could not transport the

200 chemical from one place to the other within the body. possibly have different affinities for the protein. Second, the speciation of ionizable functional groups of the protein is also $\mathrm{pH}$ dependent, which alters the overall charge of the protein and can influence the interactions with IOCs. Third, serum albumin changes its conformation depending

207 on the $\mathrm{pH}$ of the solution, ${ }^{9}$ possibly changing the binding site structure. Salt type and 208 concentration of the medium is another crucial factor that has to be considered, because chloride, 209 for example, is reported to compete with warfarin ${ }^{17}$ and fatty acids ${ }^{18}$ (both are anionic chemicals) 210 for the high affinity binding sites of serum albumin.

211 For 2,6-dichlorobenzoic acid the observed $\mathrm{pH}$ dependence was relatively small; $\log K_{\mathrm{BSA} / \mathrm{w}}$ is

$2121.86,1.82$ and 1.72 for $\mathrm{pH} 6,7$, and 8, respectively. A former study found an increasing, a

213 decreasing, or no clear trend in $\mathrm{pH}$ dependence of BSA binding for perfluoroalkyl acids of 214 different chain lengths. ${ }^{7}$ These results indicate that changes in $\mathrm{pH}$ have different effects on the 215 sorption behavior, depending on the chemicals. In contrast to the relatively small $\mathrm{pH}$ dependence 216 observed here, the results for the measurements at different salt concentrations for 2,6217 dichlorobenzoic acid show a clear competition effect (Figure S2, SI). An increase of the $\mathrm{Cl}^{-}$ 218 concentration by a factor of 50 decreases $K_{\mathrm{BSA} / \mathrm{w}}$ by a factor of 17 (i.e., $1.2 \log$ units). Moreover, $219 K_{\mathrm{BSA} / \mathrm{w}}$ of 2,6-dichlorobenzoic acid was determined to be 1.8 times higher at $163 \mathrm{mM} \mathrm{SO}_{4}^{2-}$ than 220 at $500 \mathrm{mM} \mathrm{Cl}^{-}$(i.e., at the same ionic strength), which indicates that the type of competing ion

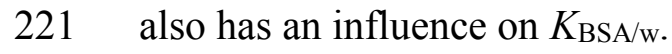


222 While further research is clearly needed to fully understand $\mathrm{pH}$ and salt dependence of $K_{\mathrm{BSA} / \mathrm{w}}$

223 for IOCs, the results obtained do support the initial statement that $\mathrm{pH}$ and salt concentration

224 should be controlled to assure comparability of data. As a consequence, all data discussed in the

225 following sections were measured with a buffer of the same composition, as described in the

226 method section.

227 BSA-water partition coefficients

228 BSA-water partition coefficients $\left(K_{\mathrm{BSA} / \mathrm{w}}\right)$ were successfully measured for 45 anionic and 4

229 cationic organic chemicals. The determined $\log K_{\mathrm{BSA} / \mathrm{w}}$ range from 0.97 to 5.27 (Table 1).

230 Table 1. Determined logarithmic BSA-water partition coefficients $\left(\log K_{\mathrm{BSA} / \mathrm{w}}\right)$ at $37^{\circ} \mathrm{C}(\mathrm{pH} 7.4$

231 in HBSS with $10 \mathrm{mM}$ Tris). Data are all for ionic species.

\begin{tabular}{lcc}
\hline Test chemical & $\begin{array}{c}\text { log } \mathbf{K S A} / \mathbf{w} \\
{[\mathbf{L} / \mathbf{k g}]}\end{array}$ & SD \\
\hline Benzoic acids & & \\
benzoic acid & 2.23 & 0.04 \\
2-chlorobenzoic acid & 1.84 & 0.02 \\
3-chlorobenzoic acid & 3.22 & 0.02 \\
4-chlorobenzoic acid & 3.21 & 0.02 \\
3,4-dichlorobenzoic acid & 4.06 & 0.02 \\
2,6-dichlorobenzoic acid & 1.65 & 0.02 \\
4-fluorobenzoic acid & 2.84 & 0.03 \\
4-nitrobenzoic acid & 2.66 & 0.02 \\
4-bromobenzoic acid & 3.48 & 0.02 \\
4-methylbenzoic acid & 2.67 & 0.02 \\
2-methylbenzoic acid & 1.99 & 0.03 \\
4-ethylbenzoic acid & 3.03 & 0.04 \\
4-butylbenzoic acid & 3.73 & 0.02 \\
4-hexylbenzoic acid & 4.23 & 0.02 \\
2,4,6-trimethylbenzoic acid & 2.26 & 0.03 \\
2-cyclohexylbenzoic acid & 3.55 & 0.04 \\
Naphthoic acids & & \\
2-naphthoic acid & 4.36 & 0.07 \\
2-naphthaleneacetic acid & 4.77 & 0.05 \\
1-naphthoic acid & 2.81 & 0.02
\end{tabular}


1-naphthaleneacetic acid

$\begin{array}{ll}3.43 & 0.03\end{array}$

4-fluoro-1-naphthoic acid

$3.46 \quad 0.01$

1-bromo-2-naphthoic acid

$4.02 \quad 0.03$

2-methoxy-1-naphthoic acid

$2.16 \quad 0.01$

2-ethoxy-1-naphthoic acid

$2.66 \quad 0.02$

3-methoxy-2-naphthoic acid

$2.86 \quad 0.01$

Phenoxy acids

2-phenoxyacetic acid

$\begin{array}{ll}2.53 & 0.07\end{array}$

2,4-dichlorophenoxyacetic acid

$3.28^{\mathrm{a}} \quad 0.10$

4-(2,4-dichlorophenoxy)butyric acid

$4.12 \quad 0.03$

2,4,5-trichlorophenoxyacetic acid

$3.83 \quad 0.02$

mecoprop $^{b}$

$3.74 \quad 0.06$

Arylpropionic acids

ketoprofen $^{\mathrm{b}}$

$3.31 \quad 0.01$

ibuprofen $^{\text {b }}$

$3.91 \quad 0.02$

fenoprofen $^{\mathrm{b}}$

$3.92 \quad 0.02$

$\alpha, 4$-dimethylphenylacetic acid ${ }^{\mathrm{b}}$

$3.00 \quad 0.03$

Phenols

pentachlorophenol

bromoxynil

$\begin{array}{ll}5.27 & 0.10\end{array}$

$5.18 \quad 0.06$

Coumarines

coumachlor

$3.41 \quad 0.02$

coumafuryl

$2.84 \quad 0.02$

Others

mefenamic acid

$\begin{array}{ll}4.36 & 0.02\end{array}$

sulcotrione

$1.72 \quad 0.06$

$\underline{\text { Sulfonates }}$

4-ethylbenzenesulfonate $\quad 3.17^{\mathrm{a}} \quad 0.03$

4-n-octylbenzenesulfonate $\quad 4.84 \quad 0.02$

2,4,6-trimethylbenzenesulfonate $\quad 4.23 \quad 0.05$

$\begin{array}{lll}\text { 4-bromobenzenesulfonate } & 3.32 & 0.04\end{array}$

naphthalene-2-sulfonate $\quad 4.71 \quad 0.04$

\section{Cations}

(S)-(-)-propranolol $\quad 1.43 \quad 0.05$

$\begin{array}{lll}\text { alprenolol }^{\mathrm{b}} & 1.02 & 0.07\end{array}$

$\begin{array}{lll}\text { imipramine } & 1.58 & 0.09\end{array}$

$\begin{array}{lll}\text { verapamil }^{\mathrm{b}} & 0.97^{\mathrm{a}} & 0.13\end{array}$

${ }^{\mathrm{a}} \log K_{\mathrm{BSA} / \mathrm{w}}$ are taken from our previous work ${ }^{16}$

b Chiral compounds. Because no information on the 
enantiomeric composition was available, we assumed the racemic mixture.

232 The organic anions measured in this study tended to show high affinities for BSA. Given that 233 serum albumin is present with a fraction of $2.9 \mathrm{vol} \%{ }^{19}$ and is the dominant sorption phase in

234 blood plasma, the majority of the anions tested (38 out of 45) are expected to be more than $90 \%$ 235 bound to serum albumin in plasma. Even the smallest anion in the dataset (benzoic acid) binds

236 strong enough to perform a binding experiment. In contrast, binding of the tested cations to BSA

237 was often too weak to be measureable (i.e., fraction bound $<20 \%$ in our dialysis experiments)

238 and $\log K_{\mathrm{BSA} / \mathrm{w}}$ was finally only determined for four cationic chemicals. Organic cations that

239 could not be measured because of too weak sorption include serotonin, (S)-(-)-nicotine, 240 dibenzylamine and different quaternary ammonium compounds (e.g., difenzoquat and

241 benzyldimethyloctylammonium). The finding that anions tend to be much stronger bound to

242 serum albumin than cations is in accordance with previous studies, ${ }^{20}$ although this relationship

243 appears not to be a strict rule, as some cationic drugs have been reported to show similar strong

244 binding as anions to serum albumin. ${ }^{21}$ Because only four cationic chemicals were successfully

245 measured in this study, the discussions below focus on the partitioning data for anions.

\section{Influences of molecular structure on $\log K_{\mathrm{BSA} / \mathrm{w}}$}

247 From the dataset presented in Table 1, it can be seen that addition of a $\mathrm{CH}_{2}$ group or a longer

248 chain to the molecule increases $\log K_{\mathrm{BSA} / \mathrm{w}}$ by, on average, 0.28-0.62 log units per $\mathrm{CH}_{2}$ unit

249 (Table S7, SI). For example, a linear increase of $\log K_{\mathrm{BSA} / \mathrm{w}}$ was measured for a homologous

250 series of 4-alkylbenzoic acids with a slope of $0.31 \log$ units $/ \mathrm{CH}_{2}$ (Figure S1, SI). Similarly, log

$251 K_{\mathrm{BSA} / \mathrm{w}}$ increases from 4.36 for 2-naphthoic acid to 4.77 for 2-naphthaleneacetic acid, from 2.81

252 for 1-naphthoic acid to 3.43 for 1-naphthaleneacetic acid and from 2.16 for 2-methoxy-1-

253 naphthoic acid to 2.66 2-ethoxy-1-naphthoic acid (for more examples, see Table S7, SI). A 
254 similar increase of $\log K_{\mathrm{BSA} / \mathrm{w}}$ with $\mathrm{CH}_{2}$ was observed for neutral compounds before (0.35-0.50

$255 \log$ units $\left./ \mathrm{CH}_{2}\right) \cdot{ }^{14} \mathrm{~A}$ consistent increase of a partition coefficient with the addition of $\mathrm{CH}_{2}$ unit is

256 common for neutral compounds in many systems including solvent-water partition systems in

257 general. ${ }^{22}$ Thus, in this regard, the observed sorption behavior of IOCs to BSA is generally in

258 line with what we see with neutral compounds (although the observed range of $\mathrm{CH}_{2}$ influences

259 on $\log K_{\mathrm{BSA} / \mathrm{w}}$ for IOCs is somewhat broader than usual for neutral compounds).

260 An interesting outcome of this work is that, for a series of anionic substituted benzenes and 261 naphthalenes measured in this study, a remarkably high influence of the substitution pattern of 262 the molecules on BSA binding was found. As presented in Figure 1A, data for benzoic acids 263 show that substitution in direct vicinity to the charged carboxylate group lowers the partition 264 coefficient substantially. For example, chlorinated benzoic acids show the following trends: 3265 chlorobenzoic acid and 4-chlorobenzoic acid have similar partition coefficients (log $K_{\mathrm{BSA} / \mathrm{w}}$ is 2663.22 and 3.21, respectively), while $\log K_{\mathrm{BSA} / \mathrm{w}}$ for 2-chlorobenzoic acid is only 1.84 (Figure 1A).

267 For the two constitutional isomers, 3,4-dichlorobenzoic acid and 2,6-dichlorobenzoic acid, a log $268 K_{\mathrm{BSA} / \mathrm{w}}$ of 4.06 and 1.65 was determined, respectively, which means a difference of more than 269 two orders of magnitude. A similar trend was found for methylated benzoic acids: $\log K_{\mathrm{BSA} / \mathrm{w}}$ is 2702.67 for 4-methylbenzoic acid, but only 1.99 for 2-methylbenzoic acid. It is also interesting that $271 \log K_{\mathrm{BSA} / \mathrm{w}}$ of 2,4,6-trimethylbenzoic acid is just 2.26, although it has two carbon atoms more 272 than 4-methylbenzoic acid (2.67). These consistent decreases of $K_{\mathrm{BSA} / \mathrm{w}}$ upon ortho-substitutions 273 on benzoic acid suggest steric effects on the BSA binding, e.g., reduced accessibility to binding 274 sites. For the chlorinated benzoic acids, the observed effects of substitution position could 275 partially be non-steric, because strongly electron-withdrawing $\mathrm{Cl}$ has various influences on 276 molecular properties of aromatic acids, which are reflected by, e.g., solvent-water partition 
277 coefficients $^{23}$ and $\mathrm{p} K_{\mathrm{a}}$ of their neutral species. For methylated benzoic acids the effect is 278 probably only steric. To obtain an additional insight, 3D structures of the substituted benzoic 279 acid molecules were optimized using the quantum chemical software Turbomole (Figures S3280 S18, SI). From these structures it can be seen that ortho-substituted benzoic acids (i.e. 2281 chlorobenzoic acid, 2,6-dichlorobenzoic acid, 2-methylbenzoic acid and 2,4,6-trimethylbenzoic 282 acid) show a twisted carboxylate group, i.e., the two oxygen atoms of the carboxylate are aligned 283 perpendicular to the benzene ring, whereas the other benzoic acids show the parallel 284 conformation. This structural feature could be related to the lower affinities of the vicinal 285 substituted benzoic acids for BSA. Note that the steric effect discussed here was indicated for 286 benzoic acids but not for sulfonates (for further discussion see the last paragraph of this section).

A

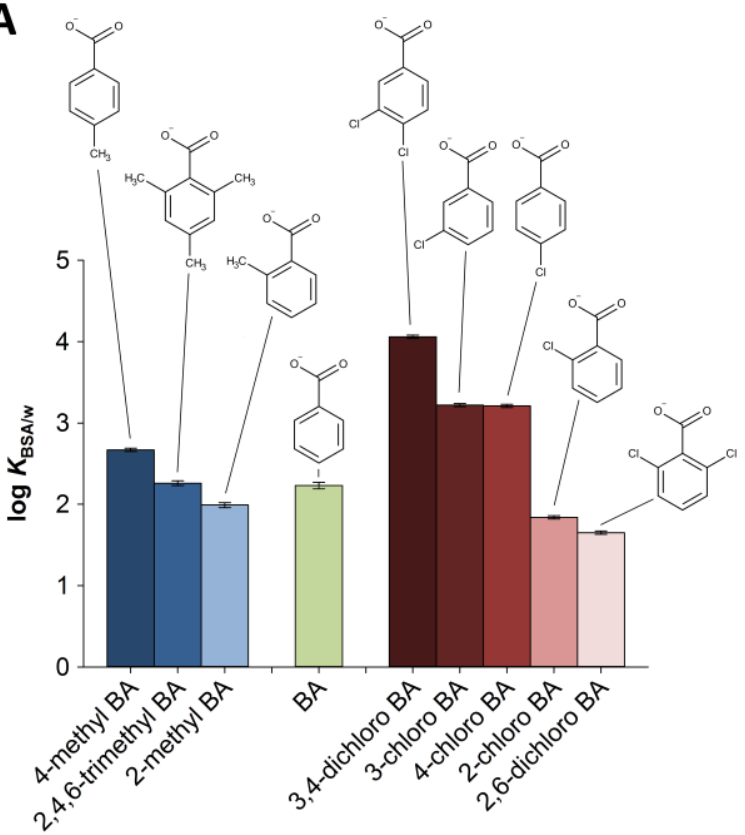

B

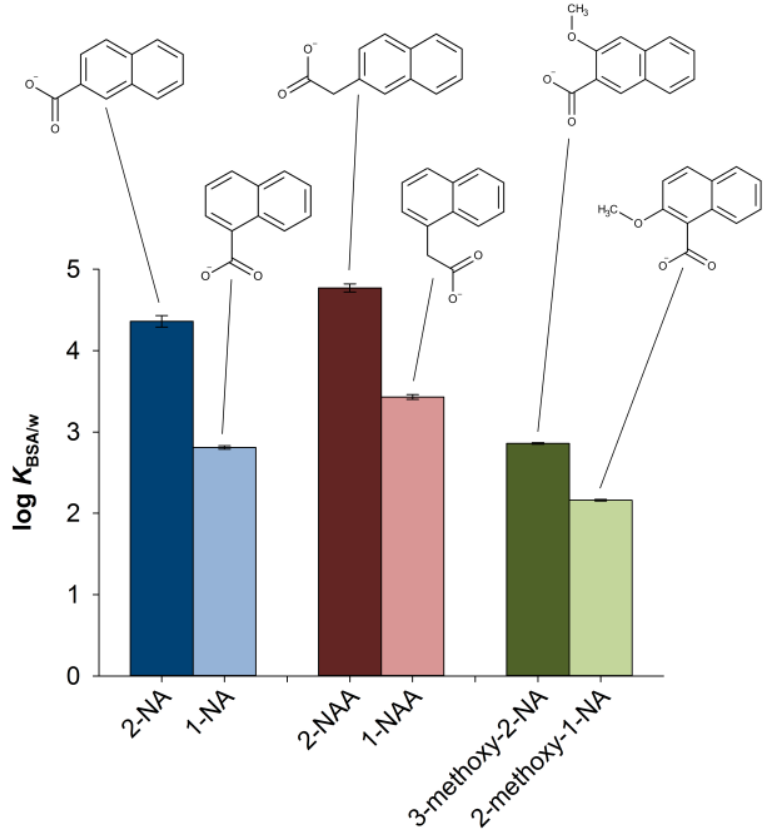

288 Figure 1. Influence of substitution position on $\log K_{\mathrm{BSA} / \mathrm{w}}$ and observed steric effects; BA 289 benzoic acid, NA - naphthoic acid, NAA - naphthaleneacetic acid. 
Another notable finding is that 1-naphthoic acids consistently have much lower BSA-water 291 partition coefficients than corresponding 2-naphthoic acids (Figure 1B). The determined log $292 K_{\mathrm{BSA} / \mathrm{w}}$ of 1-naphthoic acid is 2.81, while it is 4.36 for 2-naphthoic acid; 1-naphthaleneacetic acid 293 and 2-naphthaleneacetic acid have $\log K_{\mathrm{BSA} / \mathrm{w}}$ of 3.43 and 4.77, respectively and 2-methoxy-1294 naphthoic acid and 3-methoxy-2-naphthoic acid of 2.16 and 2.86, respectively. The difference 295 might be explained by the 3D shape of these chemicals, because 2-naphthoic acids are more 296 linear, whereas the 1-naphthoic acids have a more bulky structure.

297 Stereoselectivity is often reported for serum albumin in the literature. ${ }^{24-26}$ For chiral drugs 298 binding of the different enantiomers to human serum albumin normally differs only up to a factor 299 of 1.5. ${ }^{24,27}$ Larger differences in serum albumin binding were found for specific isomers, e.g., the 300 amino acid tryptophan, for which the L chiral form is reported to bind 10 - 100 times more to 301 serum albumin than the D form. ${ }^{25,28,29}$ By comparison, the high influences of the 3D structure on 302 the partitioning of benzoic and naphthoic acids to BSA that were found in this study are 303 remarkable, considering the generally broad specificity of the albumin binding. In general, 304 organic anions are believed to bind to a hydrophobic pocket of serum albumin with additional 305 electrostatic interactions between the negative charge of the chemical and the positive charge of 306 a lysyl- or arginyl residue. ${ }^{9}$ Possibly, the accessibility of such a binding site is hampered for 307 some of the chemicals tested in this study due to their 3D shape.

308 In the dataset, we included four pairs of chemicals that have the same non-ionic substructure 309 but different charged functional groups (i.e., sulfonate vs carboxylate): 4-ethylbenzenesulfonate 310 and 4-ethylbenzoic acid, naphthalene-2-sulfonate and 2-naphthoic acid, 4311 bromobenzenesulfonate and 4-bromobenzoic acid, and 2,4,6-trimethylbenzenesulfonate and 312 2,4,6-trimethylbenzoic acid. For the first three pairs, the type of the charged functional group has 
313 only a minor influence on $\log K_{\mathrm{BSA} / \mathrm{w}}(0.14-0.35 \log$ units). However, a large difference (1.97 log

314 units) was found between 2,4,6-trimethylbenzenesulfonate and 2,4,6-trimethylbenzoic acid,

315 which cannot easily be explained. As already mentioned above, 2,4,6-trimethylbenzoic acid has

316 two methyl substitutions in direct vicinity of the charged group and its $K_{\mathrm{BSA} / \mathrm{w}}$ value is even

317 lower than that of benzoic acid most likely due to a steric effect. In contrast, 2,4,6-

318 trimethylbenzenesulfonate appears not to experience such a steric effect, as is indicated by the

$319 K_{\mathrm{BSA} / \mathrm{w}}$ value being greater than that of 4-ethylbenzenesulfonate. Corroborating this interpretation,

320 the sulfonate group of 2,4,6-trimethylbenzenesulfonate optimized by Turbomole shows no

321 structural difference compared to 4-ethylbenzenesulfonate (Figure S19, SI).

\section{Comparison with other partition coefficients}

323 As a first step towards developing a predictive model for partitioning of organic ions to serum

324 albumin, we plotted $\log K_{\mathrm{BSA} / \mathrm{w}}$ determined in this study against various other partition

325 coefficients that can be derived more easily than $\log K_{\mathrm{BSA} / \mathrm{w}}$ itself (Figures 2 and 3).

326 First, the data are compared with logarithmic octanol-water partition coefficients of the neutral

327 species of the test chemicals $\left(\log K_{\text {ow }}\right.$ (neutral)), although we are not hypothesizing that there is a

328 mechanistic relationship between $\log K_{\mathrm{BSA} / \mathrm{w}}$ and $\log K_{\text {ow }}$ (neutral). $\log K_{\text {ow }}$ (neutral) data are

329 readily available from the literature and many environmental models are based on correlations

330 with $\log K_{\text {ow }}$ (neutral), although it has been shown that such models are purely on an empirical

331 basis and can be inaccurate even for neutral chemicals. ${ }^{2}$ All $\log K_{\text {ow }}$ values shown in Figure 2 are

332 taken from the EPI-Suite data base (version 4.1) provided by the U.S. Environmental Protection

333 Agency. If no experimental $\log K_{\text {ow }}$ (neutral) was available, the value calculated from EPISuite

334 was used instead (Table S8, SI). For comparison, BSA binding data for 83 neutral chemicals

335 from Endo et al. ${ }^{14}$ are included in Figure 2. For the neutral chemicals, there is a relatively weak 
336 but clear positive trend between $\log K_{\mathrm{BSA} / \mathrm{w}}$ and $\log K_{\mathrm{ow}}\left(R^{2}=0.75\right) \cdot{ }^{14}$ In contrast, for the organic 337 anions and cations of this study, there is no overall trend $\left(R^{2}=0.13\right.$ for all anions). The high 338 scatter appears to result from several reasons. First, according to EPI-Suite, sulfonates have 339 extremely small $\log K_{\text {ow }}$ (neutral), except for naphthalene-2-sulfonate, which is the only 340 sulfonate for which an experimental $\log K_{\text {ow }}$ (neutral) was available. The reliability of the $\log$ $341 K_{\text {ow }}$ (neutral) calculated by EPI-Suite for sulfonates is unclear, because experimental $\log K_{\text {ow }}$ for 342 neutral species of sulfonates are difficult to measure. Second, for the cationic test chemicals, the 343 relationship between $\log K_{\mathrm{BSA} / \mathrm{w}}$ (ion) and $\log K_{\mathrm{ow}}$ (neutral) appears to be substantially different 344 from that of anions. Third, while $\log K_{\text {ow }}$ (neutral) captures the increase of $\log K_{\mathrm{BSA} / \mathrm{w}}$ by addition 345 of $\mathrm{CH}_{2}$ increments, the other trends that were found in the dataset for benzoic and naphthoic 346 acids of this study (e.g., influence of substitution position and differences between 1- and 2347 naphthoic acids) cannot be depicted with $\log K_{\text {ow }}$ (neutral) (see also Figure S20A, SI).

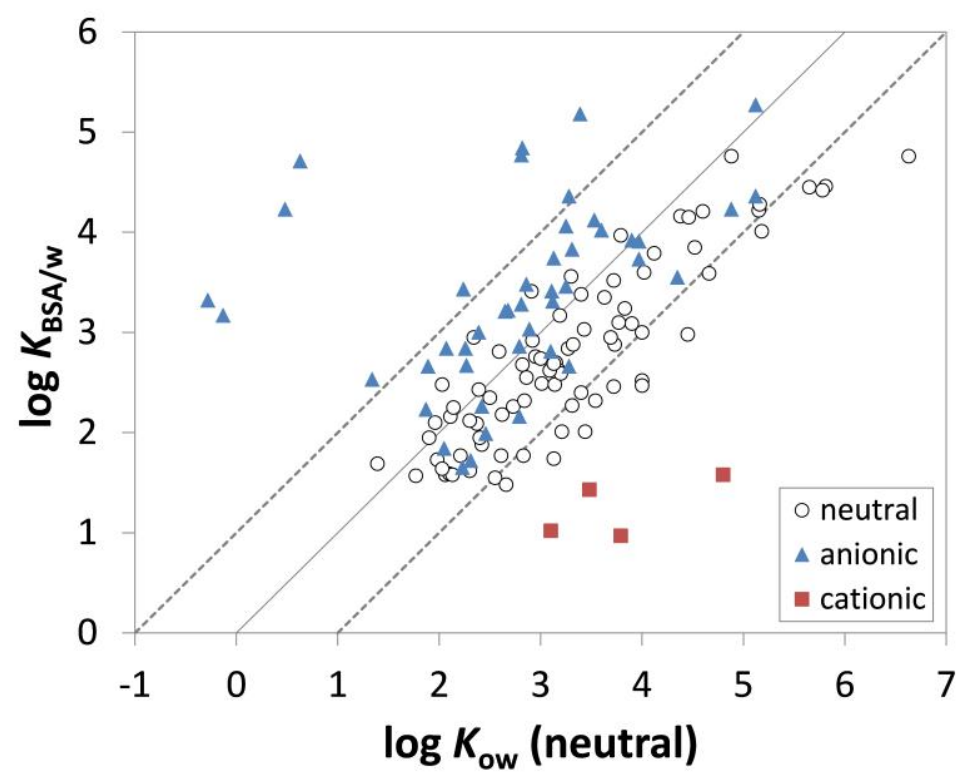

349 Figure 2. Comparison of determined $\log K_{\mathrm{BSA} / \mathrm{w}}$ (ion) with octanol-water partition coefficients of 350 the neutral species $\left(\log K_{\text {ow }}\right.$ (neutral)). 
351 We also plotted the experimental $\log K_{\mathrm{BSA} / \mathrm{w}}$ values of this study against other partition 352 coefficients of the ionic species (Figure 3), expecting a better correlation compared to $\log K_{\text {ow }}$ 353 (neutral), because these partition coefficients directly reflect physicochemical properties of the 354 ionic species of the test chemicals. For the 45 anions measured in this study, theoretical $\log K_{\text {ow }}$ 355 of the ionic species ( $\log K_{\text {ow }}$ (ion)) were calculated using the quantum chemically based software 356 COSMOtherm. ${ }^{30}$ For ionic chemicals, COSMOtherm calculates single-ion partition coefficients 357 at infinite dilution that are inaccessible by experiment (because counter-ions also partition in real 358 systems). While a positive trend does exist, the correlation of measured $\log K_{\mathrm{BSA} / \mathrm{w}}$ with 359 calculated $\log K_{\text {ow }}$ (ion) is rather weak $\left(R^{2}=0.37\right.$, Figure $\left.3 \mathrm{~A}\right)$. We also tried other solvents than 360 octanol such as methanol, acetone, and hexadecane, but none of them resulted in a better 361 correlation. Similar to $\log K_{\text {ow }}$ (neutral), $\log K_{\text {ow }}$ (ion) only accounts for the increase of $\log$ $362 K_{\mathrm{BSA} / \mathrm{w}}$ with the number of $\mathrm{CH}_{2}$ groups, but not at all for the observed differences between 1- and 363 2-naphthoic acids and the influence of substitution position (Figure S20B in SI). According to 364 COSMOtherm calculations, $\log K_{\text {ow }}$ (ion) for the structural isomers are rather similar (difference $365 \leq 0.3 \log$ units). The fact that sulfonates and benzoic acids with the same non-ionic substructure 366 show very similar $\log K_{\mathrm{BSA} / \mathrm{w}}$ (ion) is captured correctly by $\log K_{\mathrm{ow}}$ (ion) (predicted difference 367 between benzoic acids and sulfonates $0.08-0.32 \log$ units). However, the large difference 368 between 2,4,6-trimethylbenzenesulfonate and 2,4,6-trimethylbenzoic acid is not predicted by log $369 K_{\text {ow }}$ (ion) (measured difference $1.97 \log$ units, predicted difference only $0.19 \log$ units). 

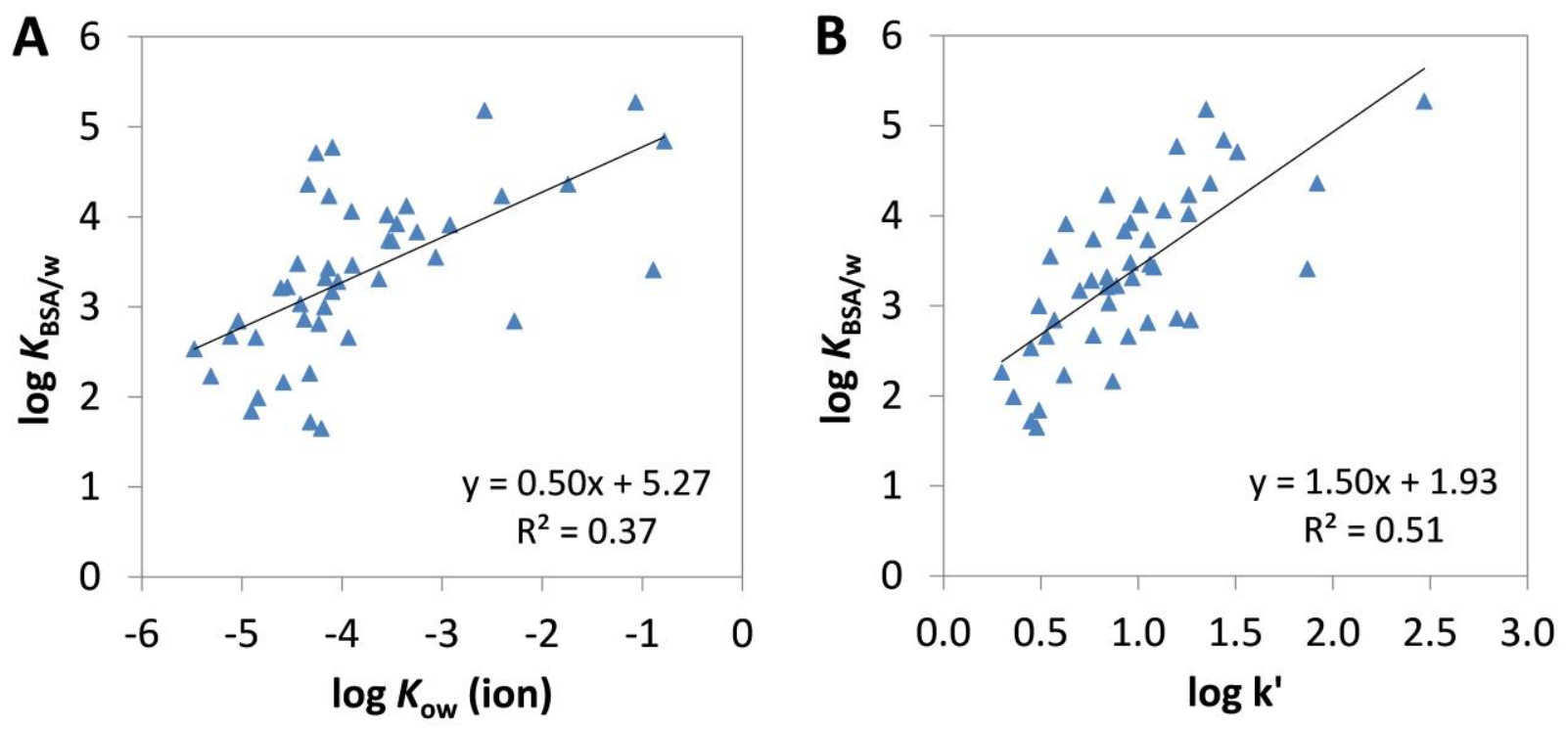

371 Figure 3. Correlation of determined $\log K_{\mathrm{BSA} / \mathrm{w}}$ for anions with (A) calculated octanol-water 372 partition coefficients of the ionic species $\left(\log K_{\mathrm{ow}}(\mathrm{ion})\right)$ and (B) measured retention factors on a 373 weak anion exchange column $\left(\log \mathrm{k}^{\prime}\right)$.

374 Additionally, in Figure 3B the data for anions are compared with measured logarithmic 375 retention factors on a weak anion exchange column (log k', Luna NH2 column, Phenomenex, 376 unpublished in-house data, see Table S8 in SI). The increase of $\log K_{\mathrm{BSA} / \mathrm{w}}$ by addition of $\mathrm{CH}_{2}$ 377 increments is correctly depicted by $\log \mathrm{k}^{\prime}$ and also the observed steric effects are captured better 378 by $\log$ k' (Figure S20C in SI) compared to $\log K_{\text {ow }}$ (both, neutral and ion). Nevertheless, the 379 overall correlation $\left(R^{2}=0.51\right)$ may still be too unsatisfying to be considered as a predictive 380 model.

\section{Modeling considerations}

382 The findings of this study suggest that conventional modeling approaches like correlation with $383 \log K_{\text {ow }}$ would fail to accurately predict the partitioning of IOCs to serum albumin. Descriptors 384 that are supposed to directly reflect the properties of the ionic species like calculated $\log K_{\text {ow }}$ of 
385 ionic species and measured retention factors on an ion exchange column do not show a good 386 correlation with measured $\log K_{\mathrm{BSA} / \mathrm{w}}$ either.

387 Polyparameter linear free energy relationship (PP-LFER) models as described by Endo et al. ${ }^{14}$ 388 are only applicable for neutral chemicals. However, Abraham et al. have proposed a PP-LFER 389 approach that can be used for ions as well. ${ }^{31,32}$ This PP-LFER model was fitted to the data of this 390 study, including 82 neutral chemicals from Endo et al ${ }^{14}$ (a detailed description of the PP-LFER 391 modeling can be found in the SI). The derived PP-LFER equation gives a better correlation for $392 \log K_{\mathrm{BSA} / \mathrm{w}}$ than the other approaches discussed above $\left(R^{2}=0.70, \mathrm{RMSE}=0.58\right.$ for all ionic 393 chemicals, Figure 4), but again important trends in the dataset such as the influence of 394 substitution position and differences between 1- and 2-naphthoic acids are not captured correctly 395 (Figure S20D, SI). This deficiency of the model is not surprising, because the solute descriptors 396 used for PP-LFER models incorporate only the volume of the solute, but not the specific 3D 397 structure. ${ }^{2}$ Because there may well be unknown structural effects that cause even larger errors, 398 we do not recommend a general use of the PP-LFER equation derived within this study to predict 399 sorption of organic ions to serum albumin.

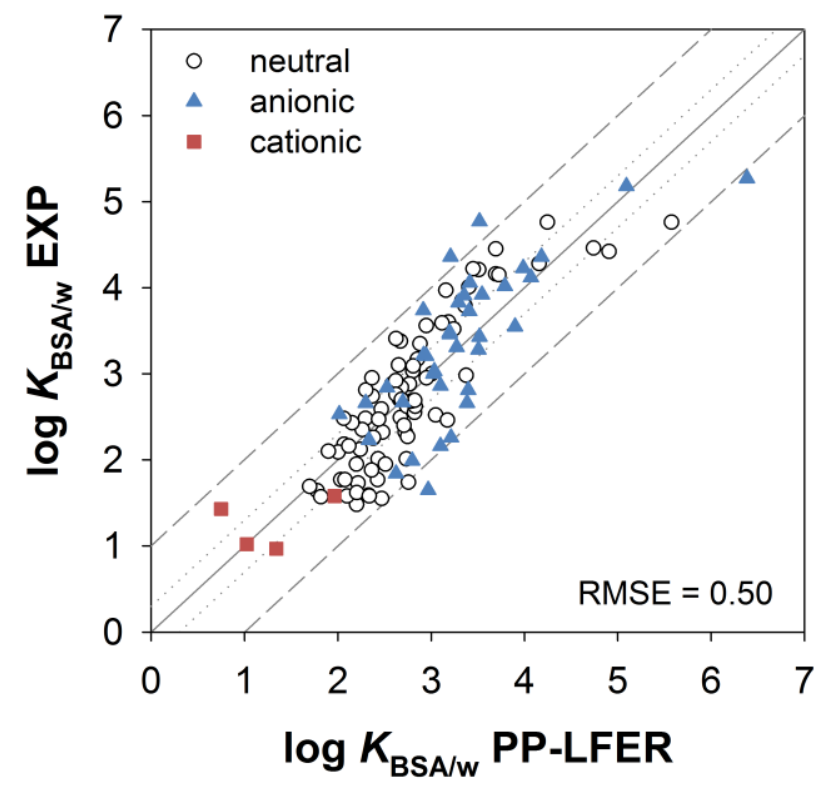


401 Figure 4. Comparison of experimentally determined BSA-water partition coefficients (log $\left.402 K_{\mathrm{BSA} / \mathrm{w}} \mathrm{EXP}\right)$ with PP-LFER fitted BSA-water partition coefficients (log $\left.K_{\mathrm{BSA} / \mathrm{w}} \mathrm{PP}-\mathrm{LFER}\right)$; solid 403 line denotes the 1:1 line, dotted and dashed lines indicate a deviation of 0.3 and $1 \log$ unit, 404 respectively.

405 In conclusion, an important reason why the conventional descriptors tested above are 406 inappropriate for modeling serum albumin binding appears to be that they cannot account for the 407 observed steric effects and the influence of the 3D structure. Thus, for a better prediction, a 408 modeling approach that correctly captures the 3D structure effects needs to be applied. There is 409 an ongoing effort in our research group to use 3D quantitative structure activity relationships 410 (3D-QSARs) for modeling serum albumin binding constants, which will be reported soon.

411 ASSOCIATED CONTENT

412 Supporting Information. Further information on the test chemicals, instrumental analysis and 413 PP-LFER modeling; 21 additional figures (including 3D structures for selected chemicals) and 9 414 additional tables. This material is available free of charge via the Internet at http://pubs.acs.org.

\section{AUTHOR INFORMATION}

\section{Corresponding Author}

417 * Tel: ++81-6-6605-2763, E-mail: satoshi.endo@urban.eng.osaka-cu.ac.jp

\section{ACKNOWLEDGMENT}

419 We thank Todd Gouin and Richard Cubberley from the Safety \& Environmental Assurance 420 Centre (SEAC), Unilever (Colworth, UK) and Nadin Ulrich at the UFZ, Leipzig for facilitating 421 the LC-MS measurements. Special thanks go to Andrea Pfennigsdorff for her assistance in 
422 laboratory work. Satoshi Endo acknowledges the support by the MEXT/JST Tenure Track

423 Promotion Program.

\section{References}

426 1. Endo, S.; Escher, B. I.; Goss, K. U., Capacities of Membrane Lipids to Accumulate 427 neutral Organic Chemicals. Environ. Sci. Technol. 2011, 45, 5912-5921.

428 2. Endo, S.; Goss, K.-U., Applications of Polyparameter Linear Free Energy Relationships 429 in Environmental Chemistry. Environ. Sci. Technol. 2014, 48, (21), 12477-12491.

$430 \quad 3 . \quad$ Poole, C. F.; Ariyasena, T. C.; Lenca, N., Estimation of the environmental properties of 431 compounds from chromatographic measurements and the solvation parameter model. J. 432 Chromatogr. A 2013, 1317, 85-104.

433 4. Bittermann, K.; Spycher, S.; Endo, S.; Pohler, L.; Huniar, U.; Goss, K.-U.; Klamt, A., 434 Prediction of Phospholipid-Water Partition Coefficients of Ionic Organic Chemicals Using the 435 Mechanistic Model COSMOmic. J. Phys. Chem. B 2014, 118, (51), 14833-14842.

436 5. Armitage, J. M.; Arnot, J. A.; Wania, F.; Mackay, D., Development and evaluation of a 437 mechanistic bioconcentration model for ionogenic organic chemicals in fish. Environ. Toxicol. 438 Chem. 2013, 32, (1), 115-128.

439 6. Fasano, M.; Curry, S.; Terreno, E.; Galliano, M.; Fanali, G.; Narciso, P.; Notari, S.; 440 Ascenzi, P., The extraordinary ligand binding properties of human serum albumin. IUBMB Life $4412005,57,(12), 787-796$. 
442 7. Bischel, H. N.; MacManus-Spencer, L. A.; Zhang, C.; Luthy, R. G., Strong associations 443 of short-chain perfluoroalkyl acids with serum albumin and investigation of binding mechanisms. 444 Environ. Toxicol. Chem. 2011, 30, (11), 2423-2430.

445 8. Fanali, G.; di Masi, A.; Trezza, V.; Marino, M.; Fasano, M.; Ascenzi, P., Human serum 446 albumin: From bench to bedside. Mol. Asp. Med. 2012, 33, (3), 209-290.

447 9. Peters Jr, T., All About Albumin. Academic Press: San Diego, 1995.

448 10. Gülden, M.; Dierickx, P.; Seibert, H., Validation of a prediction model for estimating 449 serum concentrations of chemicals which are equivalent to toxic concentrations in vitro. Toxicol. 450 in Vitro 2006, 20, (7), 1114-1124.

451 11. Gülden, M.; Mörchel, S.; Tahan, S.; Seibert, H., Impact of protein binding on the 452 availability and cytotoxic potency of organochlorine pesticides and chlorophenols in vitro. 453 Toxicology 2002, 175, (1-3), 201-213.

454 12. van der Heijden, S. A.; Hermens, J. L. M.; Sinnige, T. L.; Mayer, P.; Gilbert, D.; Jonker, 455 M. T. O., Determining High-Quality Critical Body Residues for Multiple Species and Chemicals 456 by Applying Improved Experimental Design and Data Interpretation Concepts. Environ. Sci. 457 Technol. 2015, 49, (3), 1879-1887.

458 13. Endo, S.; Bauerfeind, J.; Goss, K.-U., Partitioning of Neutral Organic Compounds to 459 Structural Proteins. Environ. Sci. Technol. 2012, 46, (22), 12697-12703.

460 14. Endo, S.; Goss, K. U., Serum Albumin Binding of Structurally Diverse Neutral Organic 461 Compounds: Data and Models Chem. Res. Toxicol. 2011, 24, (12), 2293-2301. 
15. Nilsson, L. B., The bioanalytical challenge of determining unbound concentration and 463 protein binding for drugs. Bioanalysis 2013, 5, (24), 3033-3050.

464 16. Oemisch, L.; Goss, K.-U.; Endo, S., Ion exchange membranes as novel passive sampling 465 material for organic ions: Application for the determination of freely dissolved concentrations. J. 466 Chromatogr. A 2014, 1370, 17-24.

467 17. Wilting, J.; van der Giesen, W. F.; Janssen, L. H.; Weideman, M. M.; Otagiri, M.; Perrin, 468 J. H., The effect of albumin conformation on the binding of warfarin to human serum albumin. 469 The dependence of the binding of warfarin to human serum albumin on the hydrogen, calcium, 470 and chloride ion concentrations as studied by circular dichroism, fluorescence, and equilibrium 471 dialysis. J. Biol. Chem. 1980, 255, (7), 3032-3037.

472 18. Honoré, B.; Brodersen, R., Detection of carrier heterogeneity by rate of ligand dialysis: 473 Medium-chain fatty acid interaction with human serum albumin and competition with chloride. 474 Anal. Biochem. 1988, 171, (1), 55-66.

475 19. Peyret, T.; Poulin, P.; Krishnan, K., A unified algorithm for predicting partition 476 coefficients for PBPK modeling of drugs and environmental chemicals. Toxicol. Appl. 477 Pharmacol. 2010, 249, (3), 197-207.

478 20. Trainor, G. L., The importance of plasma protein binding in drug discovery. Expert Opin. 479 Drug Discov. 2007, 2, (1), 51-64.

480 21. Kragh-Hansen, U., Molecular aspects of ligand binding to serum albumin. Pharmacol. 481 Rev. 1981, 33, (1), 17-53. 
483 Constant. J. Phys. Chem. B 2003, 107, (50), 14025-14029.

484 23. Niederer, C.; Goss, K.-U., Effect of ortho-chlorine substitution on the partition behavior 485 of chlorophenols. Chemosphere 2008, 71, (4), 697-702.

486 24. Cheruvallath, V. K.; Riley, C. M.; Narayanan, S. R.; Lindenbaum, S.; Perrin, J. H., A 487 quantitative circular dichroic investigation of the binding of the enantiomers of ibuprofen and 488 naproxen to human serum albumin. J. Pharm. Biomed. Anal. 1997, 15, (11), 1719-1724.

489 25. Lagercrantz, C.; Larsson, T.; Denfors, I., Stereoselective binding of the enantiomers of 490 warfarin and trytophan to serum albumin from some different species studied by affinity 491 chromatography on columns of immobilized serum albumin. Comp. Biochem. Physiol., C: Comp. 492 Pharmacol. 1981, 69, (2), 375-378.

493 26. Ascoli, G. A.; Domenici, E.; Bertucci, C., Drug binding to human serum albumin: 494 Abridged review of results obtained with high-performance liquid chromatography and circular 495 dichroism. Chirality 2006, 18, (9), 667-679.

496 27. Oravcova', J.; Böhs, B.; Lindner, W., Drug-protein binding studies new trends in 497 analytical and experimental methodology. J. Chromatogr. B Biomed. Sci. Appl. 1996, 677, (1), $498 \quad 1-28$.

499 28. McMenamy, R. H.; Oncley, J. L., The Specific Binding of 1-Tryptophan to Serum 500 Albumin. J. Biol. Chem. 1958, 233, (6), 1436-1447. 
29. Fielding, L.; Rutherford, S.; Fletcher, D., Determination of protein-ligand binding 502 affinity by NMR: observations from serum albumin model systems. Magn. Reson. Chem. 2005, $50343,(6), 463-470$.

504 30. Klamt, A., Conductor-like Screening Model for Real Solvents: A New Approach to the 505 Quantitative Calculation of Solvation Phenomena. J. Phys. Chem. 1995, 99, (7), 2224-2235.

507 Ionic Species from Water to Organic phases. J. Org. Chem. 2010, 75, (4), 1006-1015.

508 32. Abraham, M. H.; Zhao, Y. H., Determination of Solvation Descriptors for Ionic Species: 509 Hydrogen Bond Acidity and Basicity. J. Org. Chem. 2004, 69, (14), 4677-4685.

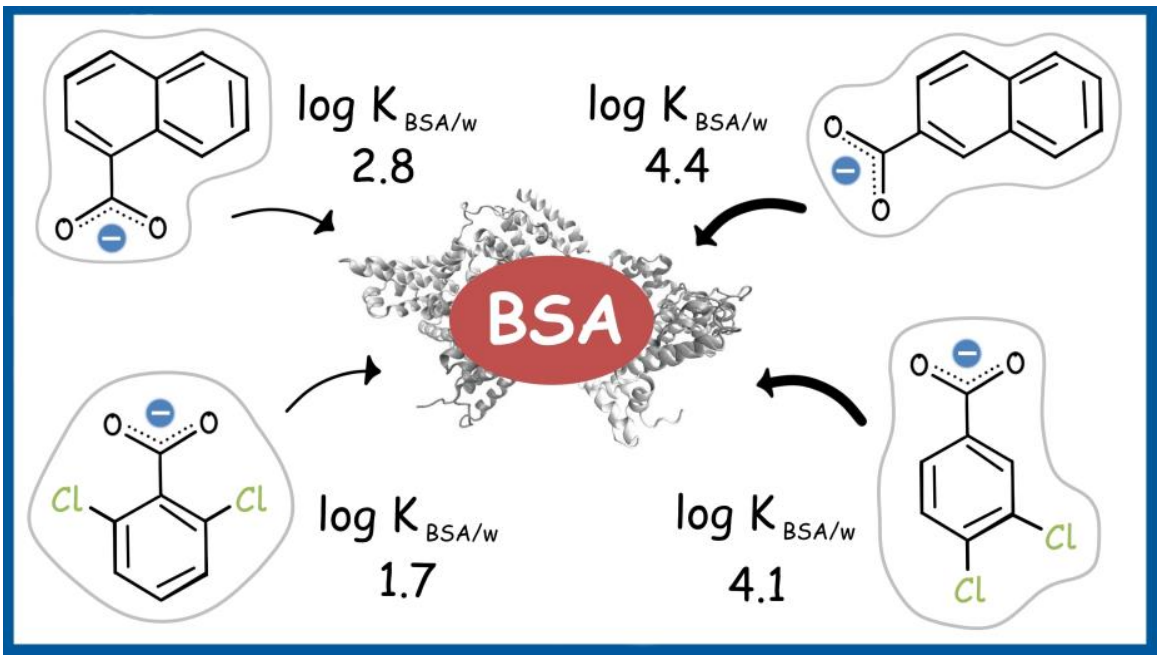

